\title{
On the Poincaré inequality for one-dimensional foliations
}

\author{
Vincent Cavalier and Daniel Lehmann
}

\begin{abstract}
Let $d$ be the degree of an algebraic one-dimensional foliation $\mathcal{F}$ on the complex projective space $\mathbb{P}_{n}$ (i.e. the degree of the variety of tangencies of the foliation with a generic hyperplane). Let $\Gamma$ be an algebraic solution of degree $\delta$, and geometrical genus $g$. We prove, in particular, the inequality $(d-1) \delta+2-2 g \geqslant \mathcal{B}(\Gamma)$, where $\mathcal{B}(\Gamma)$ denotes the total number of locally irreducible branches through singular points of $\Gamma$ when $\Gamma$ has singularities, and $\mathcal{B}(\Gamma)=1$ (instead of 0) when $\Gamma$ is smooth. Equivalently, when $\Gamma=\bigcap_{\lambda=1}^{n-1} S_{\lambda}$ is the complete intersection of $n-1$ algebraic hypersurfaces $S_{\lambda}$, we get $\left(d+n-\sum_{\lambda=1}^{n-1} \delta_{\lambda}\right) \delta \geqslant \mathcal{B}(\Gamma)-\mathcal{E}(\Gamma)$, where $\delta_{\lambda}$ denotes the degree of $S_{\lambda}$ and $\mathcal{E}(\Gamma)=2-2 g+\left(\sum_{\lambda} \delta_{\lambda}-(n+1)\right) \delta$ the correction term in the genus formula. These results are also refined when $\Gamma$ is reducible.
\end{abstract}

\section{Introduction}

In connection with the existence of first integrals, Poincaré raised the question of bounding the degree $\delta$ of an algebraic solution $\Gamma$ for an algebraic differential system $\mathcal{F}$ on the complex projective plane $\mathbb{P}_{2}$, in terms of the degree $d$ of $\mathcal{F}$. This is not possible without further conditions on $\mathcal{F}$ or on $\Gamma$. For example, Lins Neto proved in [Lin02] that the problem has no solution in the presence of dicritical singularities, i.e. of singularities through which there are infinitely many germs of separatrices (see Example 5.6 below).

In fact, the inequality $d+2-\delta \geqslant 0$ has been proved by Cerveau and Lins Neto [CL91] (see also [Soa01]) when $\Gamma$ has only nodal singularities, and by Carnicer [Car94] when the foliation has no dicritical singularity. Moreover, Brunella [Bru97] recovered Carnicer's result by observing that the negativity of the GSV-indices (see [GSV91]) is an obstruction to the above inequality, and proving that these indices are always non-negative in the non-dicritical case. Carnicer and Campillo [CC97] proved also that there exists some non-negative integer $a$, depending on conditions imposed on $\mathcal{F}$ or on $\Gamma$, such that $d+2-\delta \geqslant-a$.

In higher dimension (i.e. for one-dimensional algebraic foliations on the complex projective space $\mathbb{P}_{n}$ leaving invariant an algebraic curve $\left.\Gamma\right)$, the inequality $\left(d+n-\sum_{\lambda=1}^{n-1} \delta_{\lambda}\right) \geqslant 1$ has been proved by Soares [Soa00], when $\Gamma$ is the complete intersection $\bigcap_{\lambda=1}^{n-1} S_{\lambda}$ of $n-1$ algebraic hypersurfaces $S_{\lambda}$ of degree $\delta_{\lambda}$, under the further conditions that $\Gamma$ be smooth, and the restriction of the foliation to $\Gamma$ has non-degenerate singularities. More generally, in [Soa97, Soa00] he gave a lower bound for the degree of the algebraic foliations leaving invariant a smooth submanifold of $\mathbb{P}_{n}$, under conditions of non-degeneracy of the foliation. Also, Esteves and Kleiman [EK03] proved the inequality $(d-1)(\delta-1)-2 g \geqslant 1-r(\Gamma), r(\Gamma)$ denoting the number of globally irreducible components.

In this paper, we consider the case of curves with any kind of singularity, in any dimension. Let $\mathcal{F}$ be the one-dimensional holomorphic foliation on a holomorphic manifold $M$ defined by a

Received 22 November 2004, accepted in final form 18 May 2005.

2000 Mathematics Subject Classification 57R20, 57R25, 19E20.

Keywords: degree of an algebraic foliation, Poincaré inequality, genus formula, GSV-index, residues.

This journal is (c) Foundation Compositio Mathematica 2006. 


\section{Cavalier and D. Lehmann}

morphism $\ell: \mathcal{L} \rightarrow T M$ of holomorphic vector bundles from a line bundle $\mathcal{L}$ into $T M$. Let $\Gamma$ be a curve in $M$ (i.e. a complex analytic set of pure complex dimension one) invariant by $\mathcal{F}$. Assume that $\Gamma$ is compact and connected. The normal bundle $N_{\Gamma_{0}}$ to the non-singular part $\Gamma_{0}$ of $\Gamma$ in $M$ has a stable class which always admits a natural extension $\left[N_{\Gamma}\right]$ in the Grothendieck group $K^{0}(\Gamma)$. If $\Gamma$ is, moreover, a locally complete intersection (LCI) in $M,\left[N_{\Gamma}\right]$ may even be realized as the stable class of a natural bundle $N_{\Gamma}$ which is a natural extension of $N_{\Gamma_{0}}$ to all of $\Gamma$. (See, for instance, [LS95, LSS95] for the LCI case, and [CLS04] in general). Denote by $\Sigma=\operatorname{Sing} \Gamma \cup(\operatorname{Sing} \mathcal{F} \cap \Gamma)$ the union (made of isolated points) of the singular part of $\Gamma$ with the set of singular points of $\mathcal{F}$ which are in $\Gamma$. To each point $m_{\alpha}$ in $\Sigma$, we can associate an integer $\operatorname{GSV}_{m_{\alpha}}(\mathcal{F}, \Gamma)$, generalizing the index of Gomez-Mont et al. [GSV91], such that

$$
c_{1}\left(\left.[T M-\mathcal{L}]\right|_{\Gamma}-\left[N_{\Gamma}\right]\right) \frown[\Gamma]=\sum_{\alpha} \operatorname{GSV}_{m_{\alpha}}(\mathcal{F}, \Gamma) .
$$

In particular, we prove, when $\Gamma$ is irreducible, the inequality

$$
\sum_{\alpha} \operatorname{GSV}_{m_{\alpha}}(\mathcal{F}, \Gamma) \geqslant \mathcal{B}(\Gamma)-\mathcal{E}(\Gamma)
$$

where $\mathcal{B}(\Gamma)$ denotes the total number of locally irreducible branches through singular points of $\Gamma$ when $\Gamma$ has singularities or when $M$ is not a projective space, and $\mathcal{B}(\Gamma)=1$ (instead of 0 ) when $\Gamma$ is smooth and $M=\mathbb{P}_{n}$; and $\mathcal{E}(\Gamma)=2-2 g-c_{1}\left(\left.T M\right|_{\Gamma}-\left[N_{\Gamma}\right]\right) \frown[\Gamma]$ denotes the correction term in the genus formula ( $g$ being the geometrical genus of $\Gamma$ ).

Equivalently, we get $-c_{1}(\mathcal{L}) \frown[\Gamma]+2-2 g \geqslant \mathcal{B}(\Gamma)$.

When $\mathcal{F}$ is an algebraic foliation of degree $d$ on $\mathbb{P}_{n}$, and when $\Gamma$ is no longer assumed to be smooth, but is still the complete intersection $\bigcap_{\lambda=1}^{n-1} S_{\lambda}$ of $n-1$ algebraic hyper-surfaces $S_{\lambda}$, we get

$$
\left(d+n-\sum_{\lambda=1}^{n-1} \delta_{\lambda}\right) \delta \geqslant \mathcal{B}(\Gamma)-\mathcal{E}(\Gamma),
$$

where $\delta_{\lambda}$ and $\delta=\prod_{\lambda} \delta_{\lambda}$ denote the degree of $S_{\lambda}$ and of $\Gamma$, respectively. More generally, even when $\Gamma$ is not a LCI, we have

$$
(d-1) \delta+2-2 g \geqslant \mathcal{B}(\Gamma) .
$$

A significative example (but others are also given in the last section) arises from irreducible curves in $\mathbb{P}_{2}$, whose singularities are all 'elementary', i.e. are multiple points with local branches all smooth and having distinct tangents. Let $n_{r}$ be the number of the $r$-uple points of $\Gamma(r \geqslant 2)$. We have then $\mathcal{B}(\Gamma)=\sum_{r} n_{r} r$ when $\Gamma$ is singular, and $\mathcal{E}(\Gamma)=\sum_{r} n_{r} r(r-1)$. We therefore get

$$
(d+2-\delta) \delta \geqslant-\sum_{r} n_{r} r(r-2)
$$

These results will also be refined when $\Gamma$ is reducible.

In $\S 2$, we fix our notation and recall some basic facts. Most of them are well known. Theorem 2.4, proved in [CLS05], is the main technical tool needed in this paper for computing the various residues. Also, the definition of the normal Chern class of a complex analytic singular compact curve, necessary for writing a genus formula in $\S 4$, is recalled. For that, $K$-theory is indispensable in the non-LCI case, and enlightening in general. Note, however, that when $\Gamma$ is the complete intersection $\bigcap_{\lambda=1}^{n-1} S_{\lambda}$ of $n-1$ algebraic hypersurfaces $S_{\lambda}$ as above, it is theoretically sufficient to know ${ }^{1}$ that $N_{\Gamma}$ is the restriction of $\bigoplus_{\lambda} \mathcal{O}\left(\delta_{\lambda}\right)$ to $\Gamma$ with the notation as above; in particular, $N_{\Gamma}=\left.\mathcal{O}(\delta)\right|_{\Gamma}$ for $n=2$.

In $\S 3$, we bound from below the degree of an algebraic foliation in words of the geometrical genus of a prescribed algebraic invariant curve. This is only a first step: in the case $n=2$ for instance,

\footnotetext{
${ }^{1}$ As usual, $\mathcal{O}(k)$ will denote the $|k|$ th tensorial power of the tautological line bundle over $\mathbb{P}_{n}$ (respectively of its dual)
} if $k$ is a negative (respectively positive) integer. 


\section{On the Poincaré IneQuality For ONE-DIMENSIONAL FOLIATIONS}

we wish to get a lower-bound expressed as much as possible in words of the degree of this invariant curve and not of its genus.

This is achieved in $\S 4$, giving by the way a $K$-theoretical interpretation of the GSV-index which may be interesting in itself.

Some examples of applications are given in $\S 5$.

\section{Notation and backgrounds (without proof)}

\subsection{Index of an analytic vector field along a branch of $\Gamma$}

Let $m$ be a point of $\Gamma$, singular or not, and $\Gamma_{h}$ a local irreducible branch of $\Gamma$ through $m$ (we write briefly $m \in h)$. Let $\left(x_{1}, x_{2}, \ldots, x_{n}\right)$ be local coordinates in $M$ near $m$, such that $m$ has coordinates $(0,0, \ldots, 0)$.

Let $\varphi: \tilde{D} \rightarrow M$ be a minimal Puiseux-parametrization of $\Gamma_{h}$ such that $\varphi(0)=m$, where $\widetilde{D}$ denotes some open 2-disk in $\mathbb{C}$ centered at 0 : to each $t \in \widetilde{D}, \varphi$ associates the point $\varphi(t)$ of local coordinates $x_{i}=\varphi_{i}(t)$. After shrinking $\tilde{D}$, we can assume moreover that all points of $\varphi(\widetilde{D})$ are regular points of $\Gamma$, except perhaps the point $m$ itself. Let $D$ be a closed disk included into $\widetilde{D}$, with center 0 and boundary the circle $\partial D$.

For any holomorphic function $f$ on $\widetilde{D}$, and also for meromorphic function with pole at 0 , denote by $\nu(f)=(1 / 2 \sqrt{-1} \pi) \int_{\partial D}(d f / f)$ the order of $f$ at 0 . In particular, denote by $\mathbf{p}_{i}$ the integer $p_{i}=\nu\left(\varphi_{i}\right)$ : this number is always greater than one if $m$ is a singular point of $\Gamma$, and greater or equal to one (one at least of the $p_{i}$ being equal to 1 ) if $m$ is a regular point.

An analytic vector field $w=\sum_{i=1}^{n} a_{i}\left(\partial / \partial x_{i}\right)$ tangent to $\Gamma_{h}$ is a map $w: t \mapsto w(t)$ which, to each $t \in \widetilde{D}$, associates the vector $\sum_{i=1}^{n} a_{i}(t)\left(\partial / \partial x_{i}\right)_{\varphi(t)} \in T_{\varphi(t)} M$ such that $w(t)$ be tangent to $\Gamma_{h}$ at any point $\varphi(t)$, and such that all components $a_{i}$ are holomorphic, including at the point $t=0$. By abuse of notation, we sometimes write $w=\sum_{i=1}^{n} a_{i}(t)\left(\partial / \partial x_{i}\right)$. In particular, denote by $w_{0}=$ $\sum_{i=1}^{n} \varphi_{i}^{\prime}(t)\left(\partial / \partial x_{i}\right)$ the tangent vector field to $\Gamma_{h}$ given by the parametrization, and for any integer $r \geqslant 0$, set more generally $w_{r}=t^{r} w_{0}$.

Lemma 2.1. Let $w$ be an analytic vector field tangent to $\Gamma_{h}$. The meromorphic function $\sigma$ on $\widetilde{D}$ such that $w=\sigma w_{0}$, which might have a priori a pole at 0 , is in fact holomorphic. In other words, there is a well-defined non-negative integer $r=\nu(\sigma)$ and a unit holomorphic function $u$ of $t(u(0) \neq 0)$ such that $w=u w_{r}$.

Definition. For any analytic vector field $w=\sigma w_{0}$ tangent to $\Gamma_{h}$, the non-negative integer $r=\nu(\sigma)$ above will be denoted by

$$
\begin{aligned}
\mu_{m}\left(w, \Gamma_{h}\right) & =\nu(\sigma), \\
& =\nu\left(a_{i}\right)-p_{i}+1
\end{aligned}
$$

and will be called the 'index' of $w$ at $m$.

In particular, let $v=\sum_{i=1}^{n} A_{i}\left(\partial / \partial x_{i}\right)$ be a holomorphic vector field on $M$, defined on a neighborhood of $m$ in $M$, and such that $\varphi^{-1} v=\sum_{i=1}^{n}\left(A_{i} \circ \varphi\right)\left(\partial / \partial x_{i}\right)$ be tangent to $\Gamma_{h}$. The number $\mu_{m}\left(\varphi^{-1} v, \Gamma_{h}\right)$ does not depend on the choices of the local coordinates, of the Puiseuxparametrization (as far as it is a minimal one), and remains unchanged if $v$ is multiplied by a unit function: we sometimes write this number as $\mu_{m}\left(\mathcal{F}, \Gamma_{h}\right)$ if $v$ defines the foliation $\mathcal{F}$.

LEMma 2.2. If $v$ denotes a local holomorphic vector field on $M$, not identically zero, vanishing at $m$, and leaving $\Gamma$ invariant, we get

$$
\mu_{m}\left(\varphi^{-1} v, \Gamma_{h}\right) \geqslant 1,
$$

and $\nu\left(A_{i} \circ \varphi\right) \geqslant p_{i}$ for all $i$ such that $\varphi_{i}$ is not identically zero. 


\section{Cavalier and D. Lehmann}

\subsection{Residues for the relative $K$-theory}

Let $S=\left\{m_{1}, m_{2}, \ldots, m_{\alpha}, \ldots, m_{s}\right\}$ be a finite family of points on the complex analytic curve $\Gamma$ in the holomorphic manifold $M$. Let $\pi: \Gamma^{\prime} \rightarrow \Gamma$ be the normalization of $\Gamma$, and $\widehat{\pi}$ the composition of $\pi$ with the inclusion $\Gamma \subset M$. Denote by $\left(m_{h}^{\prime}\right)_{h}$ the points in $S^{\prime}=\pi^{-1}(S)$ : we get one point $m_{h}^{\prime}$ for any locally irreducible branch $\Gamma_{h}$ of $\Gamma$ through a point $m_{\alpha}$ of $S$, and $\pi\left(m_{h}^{\prime}\right)=m_{\alpha}$ if and only if $\Gamma_{h}$ is a branch through $m_{\alpha}$, which will be written $\alpha \in h$. Similarly, the notation $h \subset C$ will mean that the branch $\Gamma_{h}$ is included into the irreducible component $C$.

Separate all points $m_{h}^{\prime}$ by open 2-disks $\widetilde{D}_{h}$ in $\Gamma^{\prime}$ centered at $m_{h}^{\prime}$. (The restriction of $\widehat{\pi}$ to $\widetilde{D}_{\alpha, h}$ may be seen as a minimal Puiseux-parametrization of $\Gamma_{h}$, once $\widetilde{D}_{h}$ identified to a 2-disk $\widetilde{D}$ of center 0 in $\mathbb{C}$ by means of a biholomorphism). Let $D_{h}$ be a closed 2 -disk, still centered at $m_{h}^{\prime}$, and bounded by circles $\partial D_{h}$. The excision theorem in $K$-theory asserts that the family of the restrictions

$$
K\left(\Gamma^{\prime}, \Gamma^{\prime} \backslash S^{\prime}\right) \rightarrow K\left(\widetilde{D}_{h}, \widetilde{D}_{h} \backslash\left\{m_{h}^{\prime}\right\}\right)=K\left(D_{h}, \partial D_{h}\right)
$$

defines an isomorphism $K\left(\Gamma^{\prime}, \Gamma^{\prime} \backslash S^{\prime}\right) \cong \bigoplus_{h} K\left(D_{h}, \partial D_{h}\right)$. Recall also (see [Ati64]) that the data of a family $\left(P_{0}, P_{1}, \ldots, P_{k}\right)$ of vector bundles over $\Gamma^{\prime}$ and of a sequence of bundles exact above $\Gamma^{\prime} \backslash S^{\prime}$

$$
\left.\left.\left.0 \rightarrow P_{0}\right|_{\Gamma^{\prime} \backslash S^{\prime}} \rightarrow P_{1}\right|_{\Gamma^{\prime} \backslash S^{\prime}} \rightarrow \cdots \rightarrow P_{k}\right|_{\Gamma^{\prime} \backslash S^{\prime}} \rightarrow 0
$$

defines naturally an element $\theta \in K\left(\Gamma^{\prime}, \Gamma^{\prime} \backslash S^{\prime}\right)$, which is a lift of $\sum_{\lambda}(-1)^{\lambda+1} P_{\lambda} \in K\left(\Gamma^{\prime}\right)$ by the natural map $K\left(\Gamma^{\prime}, \Gamma^{\prime} \backslash S^{\prime}\right) \rightarrow K\left(\Gamma^{\prime}\right)$.

We call the 'residue' of $\theta$ the image $\operatorname{Res}(\theta)=c_{1}(\theta) \frown[\mathcal{T}, \partial \mathcal{T}]$ of the Chern class $c_{1}(\theta) \in$ $H^{2}\left(\Gamma^{\prime}, \Gamma^{\prime} \backslash S^{\prime} ; \mathbb{Z}\right)$ by the Alexander duality

$$
(\text {. }) \frown[\mathcal{T}, \partial \mathcal{T}]: H^{2}\left(\Gamma^{\prime}, \Gamma^{\prime} \backslash S^{\prime} ; \mathbb{Z}\right) \rightarrow H_{0}\left(S^{\prime} ; \mathbb{Z}\right)
$$

(where $\left.[\mathcal{T}, \partial \mathcal{T}]=\sum_{h}\left[D_{h}, \partial D_{h}\right)\right]$ denotes the fundamental class of $\left(\Gamma^{\prime}, \Gamma^{\prime} \backslash S^{\prime}\right)$ ).

Denoting by $\left(\theta_{h}\right)_{h} \in K\left(D_{h}, \partial D_{h}\right)$ the components of $\theta$ relative to the isomorphism above, we can define in the same way $\operatorname{Res}_{h}(\theta)=c_{1}\left(\theta_{h}\right) \frown\left[D_{h}, \partial D_{h}\right] \in \mathbb{Z}$.

We write $\operatorname{Res}_{m_{\alpha}}(\theta)=\sum_{h, \alpha \in h} \operatorname{Res}_{h}(\theta)$ for any $m_{\alpha} \in S$ so that $\operatorname{Res}(\theta)=\sum_{m_{\alpha} \in S} \operatorname{Res}_{m_{\alpha}}(\theta)$.

Similarly, for any irreducible component $C$ of $\Gamma$, we define the image $\theta_{C}$ of $\theta$ by the restriction $K\left(\Gamma^{\prime}, \Gamma^{\prime} \backslash S^{\prime}\right) \rightarrow K\left(C^{\prime}, C^{\prime} \backslash\left(C^{\prime} \cap S^{\prime}\right)\right)$. We set $\operatorname{Res}_{C}(\theta)=\sum_{h, h \subset C} \operatorname{Res}_{h}(\theta)$.

LEMMA 2.3. We get

$$
\left[\sum_{\lambda=0}^{k}(-1)^{\lambda+1} c_{1}\left(P_{\lambda}\right)\right] \frown\left[\Gamma^{\prime}\right]=\sum_{m_{\alpha} \in S} \operatorname{Res}_{m_{\alpha}}(\theta)
$$

and, for any $C$,

$$
\left[\sum_{\lambda=0}^{k}(-1)^{\lambda+1} c_{1}\left(P_{\lambda}\right)\right] \frown\left[C^{\prime}\right]=\sum_{h, h \subset C} \operatorname{Res}_{h}(\theta) .
$$

\subsection{Computation of the residues}

Let $D$ be a closed 2-disk centered at a point $a$ in $\mathbb{C}$, and $\xi$ the element in $K(D, \partial D)$ defined by a sequence $\left(P_{\lambda}, \beta_{\lambda}\right)_{\lambda}$ of holomorphic complex fibre bundles above $D_{a}$ whose restriction

$$
\left.\left.\left.\left.0 \rightarrow P_{0}\right|_{D \backslash\{a\}} \stackrel{\beta_{0}}{\longrightarrow} P_{1}\right|_{D \backslash\{a\}} \stackrel{\beta_{1}}{\longrightarrow} \cdots \stackrel{\beta_{k-2}}{\longrightarrow} P_{k-1}\right|_{D \backslash\{a\}} \stackrel{\beta_{k-1}}{\longrightarrow} P_{k}\right|_{D \backslash\{a\}} \rightarrow 0
$$

to $D \backslash\{a\}$ is exact.

As $D$ is contractible, one can assume that all bundles $P_{\lambda}$ are trivial and the morphism $\beta_{\lambda}$ defined by a matrix with holomorphic coefficients. As the sequence of morphisms is exact 


\section{On the Poincaré InEQUALity For ONE-DIMENSIONAL FOLIATIONS}

above $D \backslash\{a\}$, any of these matrices has a constant rank $r_{\lambda}$ above $D \backslash\{a\}$ : for any point $m \in D \backslash\{a\}$, the value $\left(\beta_{\lambda}\right)_{m}$ of $\beta_{\lambda}$ has a square submatrix of size $r_{\lambda}$ with non-vanishing determinant. As any holomorphic function defined on $D$, which vanishes at all points of a sequence in $D \backslash\{a\}$ converging to $a$, is identically zero, it is possible to choose the same lines and the same columns at any point $m$ in $D \backslash\{a\}$ for the determination of the above square submatrix: more precisely, by induction on $\lambda$ we can define, a square submatrix $\psi_{\lambda}$ of $\beta_{\lambda}$, of size $r_{\lambda}$, whose determinant $\Delta_{\lambda}$ does not vanish on $D \backslash\{a\}$, and such that $P_{\lambda}=K_{\lambda} \oplus C_{\lambda}$ when $\psi_{\lambda}: C_{\lambda} \rightarrow K_{\lambda+1}$ is interpreted as a linear map from a subbundle $C_{\lambda}$ of $P_{\lambda}$ into a subbundle $K_{\lambda+1}$ of $P_{\lambda+1}$. Denote by $\nu_{\lambda}=\nu\left(\Delta_{\lambda}\right)$ the order of the determinant $\Delta_{\lambda}$ at $a$.

TheOREM 2.4 [CLS05]. The following formula holds:

$$
c_{1}(\xi) \frown[D, \partial D]=\sum_{\lambda}(-1)^{\lambda} \nu_{\lambda}
$$

\subsection{Definition of the normal Chern classes to $\Gamma$ in the non-smooth case}

Let $\Gamma$ be a compact complex analytic curve in a holomorphic manifold $M$ of complex dimension $n$. Denote by $\mathcal{O}_{M}$ the sheaf of germs of holomorphic functions on $M$, by $\mathcal{I}$ the sheaf of ideals of the germs of holomorphic functions on $M$ vanishing on $\Gamma$, and by $\mathcal{O}_{\Gamma}$ the quotient sheaf $\mathcal{O}_{M} / \mathcal{I}$. Let $\pi: \Gamma^{\prime} \rightarrow \Gamma$ denote the normalization of $\Gamma$, and $\hat{\pi}: \Gamma^{\prime} \rightarrow M$ the composition of $\pi$ with the natural inclusion $\Gamma \subset M$. After [AH61], there exists a $\mathcal{A}$-locally free resolution of length at most 2:

$$
0 \rightarrow \underline{E}_{2}^{*} \stackrel{\lambda_{2}^{*}}{\longrightarrow} \underline{E}_{1}^{*} \stackrel{\lambda_{1}^{*}}{\longrightarrow} \underline{E}_{0}^{*} \stackrel{\lambda_{0}^{*}}{\longrightarrow} \mathcal{A} \otimes_{\mathcal{O}_{\Gamma^{\prime}}} \hat{\pi}^{-1}\left[\left(\mathcal{O}_{M} / \mathcal{I}\right) \otimes_{\mathcal{O}_{M}}\left(\mathcal{I} / \mathcal{I}^{2}\right)\right] \rightarrow 0
$$

of the sheaf $\mathcal{A} \otimes_{\mathcal{O}_{\Gamma^{\prime}}} \hat{\pi}^{-1}\left[\left(\mathcal{O}_{M} / \mathcal{I}\right) \otimes_{\mathcal{O}_{M}}\left(\mathcal{I} / \mathcal{I}^{2}\right)\right]$, where $\mathcal{A}$ denotes the sheaf of germs of $\mathbb{R}$-analytical $\mathbb{C}$-valued functions on $\Gamma^{\prime}$ and $\underline{E}_{j}^{*}$ the sheaf of germs of $\mathbb{R}$-analytical sections of some $\mathbb{R}$-analytical complex vector bundle $E_{j}^{*}$ over $\Gamma^{\prime}$. Let $E_{j}$ denote the dual of $E_{j}^{*}$.

Note that the restriction of $\left(\mathcal{I} / \mathcal{I}^{2}\right)$ to $\Gamma$ is the conormal sheaf, which coincides on $\Gamma_{0}$ with the sheaf of germs of holomorphic sections of the bundle $N_{\Gamma_{0}}^{*}$ dual to $N_{\Gamma_{0}}$. Thus, $\left[E_{0}-E_{1}+E_{2}\right] \in K^{0}\left(\Gamma^{\prime}\right)$ is an extension to all of $\Gamma^{\prime}$ of the stable class $\left[\pi^{-1} N_{\Gamma_{0}}\right]$ of $\pi^{-1} N_{\Gamma_{0}}$, and it does not depend after [AH61] on the choice of the resolution $(*)$. Moreover, $\pi$ induces a monomorphism $\pi^{*}: K^{0}(\Gamma) \rightarrow K^{0}\left(\Gamma^{\prime}\right)$, and $\left[E_{0}-E_{1}+E_{2}\right]$ belongs to its image, so that we get a well-defined element $\left[N_{\Gamma}\right] \in K^{0}(\Gamma)$ whose image by $\pi^{*}$ is $\left[E_{0}-E_{1}+E_{2}\right]$. We define the normal Chern classes of $\Gamma$ in $M$ as being those of $\left[N_{\Gamma}\right]$.

By duality, we get a sequence of $\mathbb{R}$-analytical complex vector bundles above $\Gamma^{\prime}$, exact above $\Gamma_{0}^{\prime}=\pi^{-1}\left(\Gamma_{0}\right)$

$$
\left.\left.\left.0 \rightarrow \pi^{-1}\left(N_{\Gamma_{0}}\right) \stackrel{\lambda_{0}}{\longrightarrow} E_{0}\right|_{\Gamma_{0}} \stackrel{\lambda_{1}}{\longrightarrow} E_{1}\right|_{\Gamma_{0}} \stackrel{\lambda_{2}}{\longrightarrow} E_{2}\right|_{\Gamma_{0}} \rightarrow 0 .
$$

If the length of $(*)$ is smaller than 2 , we understand that some of the $E_{i}^{*}$ may be of rank 0 .

In particular, when $\Gamma$ is a LCI in $M$, the conormal sheaf $\mathcal{I} / \mathcal{I}^{2}$ is $\left(\mathcal{O}_{M} / \mathcal{I}\right)$-locally free, and $\left(\mathcal{O}_{M} / \mathcal{I}\right)$-isomorphic to the sheaf of sections of some $\left(\mathcal{O}_{M} / \mathcal{I}\right)$-complex vector bundle $N_{\Gamma}^{*}$, whose dual $N_{\Gamma}$ is a natural extension of $N_{\Gamma_{0}}$. Therefore, in this case, we can take $E_{0}=\pi^{-1} N_{\Gamma}^{*}, E_{1}=$ $E_{2}=0$. More precisely, near each of its points $m, \Gamma$ is defined locally by a reduced equation $f=0\left(f: U \rightarrow \mathbb{C}^{n-1}\right.$ denoting some holomorphic map from an open neighborhood $U$ of $m$ in $\left.M\right)$, whose components $f_{i}(1 \leqslant i \leqslant n-1)$ generate the ideal $\left.\mathcal{I}\right|_{U}$. Moreover, the data of the local reduced equation $f=0$ for $\Gamma$ defines a local trivialization of $N_{\Gamma}$, such that $d f:\left.T M\right|_{\Gamma_{0} \cap U} \rightarrow \mathbb{C}^{n-1}$ corresponds to the natural projection $\varpi:\left.T M\right|_{\Gamma_{0}} \rightarrow N_{\Gamma_{0}}$ by this trivialization. 


\section{Cavalier and D. Lehmann}

\section{Bounding from below the degree of an algebraic foliation having a prescribed algebraic solution $\Gamma$}

Let $\ell: \mathcal{L} \rightarrow T M$ be a one-dimensional holomorphic foliation $\mathcal{F}$ on a holomorphic manifold $M$, and $\Gamma$ a compact curve in $M$ invariant by $\mathcal{F}$. Let $\pi: \Gamma^{\prime} \rightarrow \Gamma$ be the normalization of $\Gamma$. Let $\Sigma$ be the union of $\operatorname{Sing}(\Gamma)$ and of $\operatorname{Sing}(\mathcal{F}) \cap \Gamma$, and set $\Sigma^{\prime}=\pi^{-1}(\Sigma)$. Assume that $\Sigma$ is a finite family of isolated points $m_{\alpha}$. For each $m_{\alpha}$, write $\left(\Gamma_{h}\right)_{\alpha \in h}$ the family of local branches $\Gamma_{h}$ of $\Gamma$ through $m_{\alpha}$, and denote by $m_{h}^{\prime}$ the point of $\Sigma^{\prime}$ contained in the lift of $\Gamma_{h}$. Denote by $\mathcal{B}(\Gamma)$ the total number of locally irreducible branches of $\Gamma$ through singular points of $\Gamma$, when $\Gamma$ is singular or is not a projective space, and $\mathcal{B}(\Gamma)=1$, when $\Gamma$ is non-singular and $M=\mathbb{P}_{n}$.

The homomorphism $\ell$ determines an isomorphism $\pi^{-1}(\ell)$ between the restrictions of $\pi^{-1}(\mathcal{L})$ and $T \Gamma^{\prime}$ to $\Gamma^{\prime} \backslash \Sigma^{\prime}$. Thus, after $\S 2.1$, we get a lift $\zeta=\zeta(\mathcal{F}, \Gamma)$ of $\left[T \Gamma^{\prime}-\pi^{-1}(\mathcal{L})\right]$ in $K\left(\Gamma^{\prime}, \Gamma^{\prime} \backslash \Sigma^{\prime}\right)$.

For any irreducible component $C$ of $\Gamma$ when $\Gamma$ is reducible, denote by $g_{C}$ its geometric genus (i.e. the genus of the connected component $C^{\prime}=\pi^{-1}(C)$ in $\Gamma^{\prime}$ ), and by $\mathcal{B}_{C}(\Gamma)$ the total number of locally irreducible branches included into $C$ through points $m_{\alpha}$ in $\operatorname{Sing}(\Gamma) \cap C$ (such a branch will be denoted shortly by $h \subset C)$. Observe that in the reducible case, $\mathcal{B}_{C}(\Gamma)$ is always at least 1 , even if $C$ is smooth.

TheOREm 3.1. With the notation of $\S 2$, we assume that $\mathcal{F}$ is defined locally near $m_{\alpha}$ by the vector field $\sum_{i=1}^{n} A_{i}\left(\partial / \partial x_{i}\right)$ with respect to local coordinates $\left(x_{1}, \ldots, x_{n}\right)$. The following equality and inequality hold:

$$
\begin{aligned}
2-2 g_{C}-\left(c_{1}(\mathcal{L}) \frown[C]\right) & =\sum_{h, h \subset C} \mu_{m_{\alpha}}\left(\varphi^{-1} v, \Gamma_{h}\right), \\
& =\sum_{h, h \subset C} \nu\left(A_{i} \circ \varphi\right)-p_{i}+1 \\
& \geqslant \mathcal{B}_{C}(\Gamma),
\end{aligned}
$$

$i$ being any index such that $\varphi_{i}(t)$ is not identically zero along $\Gamma_{h}$.

Proof. As $v$ is the image of a trivialization of $\mathcal{L}$ by $\ell$, the restriction of $\pi^{-1}(\mathcal{L})$ (respectively $T \Gamma^{\prime}$ ) to a small disk $D_{h}$ around $m_{h}^{\prime}$ is trivialized by $\varphi^{-1} v$ (respectively $w_{0}$ ), with the notation of $\S 2$, a minimal Puiseux-parametrization $\varphi$ of $\Gamma_{h}$ being given by the composition of $\pi$ with a biholomorphism $D \rightarrow D_{h}$. Set $\varphi^{-1} v=\sigma w_{0}$. The homomorphism $\pi^{-1}(\ell)$ is then defined by the multiplication by $\sigma$, and the formula of Lemma 2.3 and Theorem 2.4 applied to $\zeta$ give the equality

$$
2-2 g_{C}-\left(c_{1}(\mathcal{L}) \frown[C]\right)=\sum_{h, h \subset C} \mu_{m_{\alpha}}\left(\varphi^{-1} v, \Gamma_{h}\right) .
$$

As $\mu_{m_{\alpha}}\left(\varphi^{-1} v, \Gamma_{h}\right) \geqslant 1$ after Lemma 2.2 , we get the conclusion of the theorem, when $\Gamma$ is singular. When $\Gamma$ is non-singular and $M=\mathbb{P}_{n}$, the foliation has necessarily at least one singular point on $\Gamma$, according to an argument of Soares [Soa97, Soa00], and we still have $\mu_{m_{\alpha}}\left(\varphi^{-1} v, \Gamma_{h}\right) \geqslant 1$ at such a point, hence the wanted formula with $\mathcal{B}_{C}(\Gamma)=1$.

Corollary to Theorem 3.1. For an algebraic foliation of degree $d$ on $\mathbb{P}_{n}$, the previous formulae become, denoting by $\delta$ the degree of $\Gamma$ and by $\delta_{C}$ the degree of $C$,

$$
d \geqslant 1+\sup _{C} \frac{2 g_{C}-2+\mathcal{B}_{C}(\Gamma)}{\delta_{C}}
$$

with the sup running through all irreducible components $C$ of $\Gamma$.

Proof. In fact, for an algebraic foliation of degree $d, \mathcal{L}$ is equal to $\mathcal{O}(1-d)$. The corollary then results from the equality

$$
c_{1}(\mathcal{O}(1-d)) \frown[C]=-(d-1) \delta_{C} .
$$




\section{On the Poincaré inequality for OnE-Dimensional Foliations}

\section{Remarks.}

(1) The right-hand term in the inequality of the corollary depends only on the curve $\Gamma$.

(2) In [EK03, Corollary 6.2], Esteves and Kleiman proved the inequality

$$
d \geqslant 1+\frac{2 g-r(\Gamma)+1}{\delta-1}
$$

where $r(\Gamma)$ denotes the number of irreducible components of $\Gamma$. Their lower-bound for $d$ may be better than ours in some cases: for instance, if $\Gamma$ is non-singular (in fact, both lower bounds coincide for $n=2$, and our lower bound coincides with that given in [Soa00] for smooth complete intersections). However, our lower bound is better when $\Gamma$ is sufficiently singular: for instance, we recover the result of [CL91] for nodal singularities.

\section{The GSV-index}

Let $\Sigma_{1}$ be the singular part $\Gamma \backslash \Gamma_{0}$ of a complex analytic curve $\Gamma$ in the holomorphic manifold $M$. We still write $\pi: \Gamma^{\prime} \rightarrow \Gamma$ the normalization of $\Gamma$, and $\widehat{\pi}$ the composition of $\pi$ with the inclusion $\Gamma \subset M$. Set $\Sigma_{1}^{\prime}=\pi^{-1}\left(\Sigma_{1}\right)$. We get an exact sequence above $\Gamma_{0}^{\prime}=\pi^{-1}\left(\Gamma_{0}\right)$

$$
\left.\left.\left.\left.0 \rightarrow T \Gamma_{0}^{\prime} \rightarrow \hat{\pi}^{-1}(T M)\right|_{\Gamma_{0}^{\prime}} \stackrel{\lambda_{0} \circ q}{\longrightarrow} E_{0}\right|_{\Gamma_{0}^{\prime}} \stackrel{\lambda_{1}}{\longrightarrow} E_{1}\right|_{\Gamma_{0}^{\prime}} \stackrel{\lambda_{2}}{\longrightarrow} E_{2}\right|_{\Gamma_{0}^{\prime}} \rightarrow 0
$$

$q$ denoting the natural projection $q:\left.T M\right|_{\Gamma_{0}} \rightarrow N_{\Gamma_{0}}$, and the $E_{i}$ defined as in $\S 2.4$.

Thus, we get a natural lift $\xi=\xi(\Gamma) \in K\left(\Gamma^{\prime}, \Gamma^{\prime} \backslash \Sigma_{1}^{\prime}\right)$ by the canonical map $K\left(\Gamma^{\prime}, \Gamma^{\prime} \backslash \Sigma_{1}^{\prime}\right) \rightarrow K\left(\Gamma^{\prime}\right)$ of $\left[\widehat{\pi}^{-1}(T M)-\pi^{-1}\left(N_{\Gamma}\right)-T \Gamma^{\prime}\right]$ in $K\left(\Gamma^{\prime}\right)$. We denote by

$$
\mathcal{E}(\Gamma)=\sum_{h} \mathcal{E}_{h}(\Gamma), \quad \mathcal{E}_{m_{\alpha}}(\Gamma)=\sum_{\alpha \in h} \mathcal{E}_{h}(\Gamma) \quad \text { and } \quad \mathcal{E}_{C}(\Gamma)=\sum_{h \subset C} \mathcal{E}_{h}(\Gamma)
$$

the residues of $-\xi$, according to the notation of $\S 2.2$.

A foliation $\ell: \mathcal{L} \rightarrow T M$ leaving $\Gamma$ invariant being given, again let $\Sigma=\Sigma_{1} \cup(\operatorname{Sing} \mathcal{F} \cap \Gamma)$ be the union of the singular part of $\Gamma$ with the set of singular points of $\mathcal{F}$ which are in $\Gamma$. Set $\Sigma^{\prime}=\pi^{-1}(\Sigma)$. The above definition of $\mathcal{E}_{m_{\alpha}}(\Gamma)$ and $\mathcal{E}_{h}(\Gamma)$ make sense for $m_{\alpha}$ belonging to $\Gamma_{0}$ (in particular, for $m_{\alpha} \in \Sigma \backslash \Sigma_{1}$ ), and $\Gamma_{h}$ being a local branch through such a point, but both are then zero, as the exact sequence $(* *)$ remains exact at the point $m_{\alpha}$. Thus, we can see $\xi(\Gamma) \in K\left(\Gamma^{\prime}, \Gamma^{\prime} \backslash \Sigma_{1}^{\prime}\right)=$ $\prod_{\pi\left(m_{h}^{\prime}\right) \in \Sigma_{1}} K\left(\Gamma^{\prime}, \Gamma^{\prime} \backslash\left\{m_{h}^{\prime}\right\}\right)$ as an element still denoted $\xi(\Gamma)$ in

$$
K\left(\Gamma^{\prime}, \Gamma^{\prime} \backslash \Sigma^{\prime}\right)=\prod_{\pi\left(m_{h}^{\prime}\right) \in \Sigma} K\left(\Gamma^{\prime}, \Gamma^{\prime} \backslash\left\{m_{h}^{\prime}\right\}\right),
$$

understanding that the $h$-components of $\xi(\Gamma)$ such that $\pi\left(m_{h}^{\prime}\right)$ belongs to $\Sigma \backslash \Sigma_{1}$ are zero.

Combining the exact sequence $\left(*_{0}\right)$ of $\S 2.4$ with $\ell$ and the natural morphism $T \Gamma^{\prime} \rightarrow \widehat{\pi}^{-1}(T M)$, we get the sequence over $\Gamma_{0}^{\prime}=\Gamma^{\prime} \backslash \Sigma^{\prime}$

$$
0 \rightarrow \hat{\pi}^{-1}(\mathcal{L}) \stackrel{\hat{\pi}^{-1}(\ell)}{\longrightarrow} \hat{\pi}^{-1}(T M) \stackrel{\lambda_{0} \circ q}{\longrightarrow} E_{0} \stackrel{\lambda_{1}}{\longrightarrow} E_{1} \stackrel{\lambda_{2}}{\longrightarrow} E_{2} \rightarrow 0,
$$

$q$ denoting the natural projection $q:\left.T M\right|_{\Gamma_{0}} \rightarrow N_{\Gamma_{0}}$. Moreover, as (***) is exact above $\Gamma^{\prime} \backslash \Sigma^{\prime}$, and as all bundles in this sequence are defined over all of $\Gamma^{\prime}$, we get an element in $K\left(\Gamma^{\prime}, \Gamma^{\prime} \backslash \Sigma^{\prime}\right)$, which is a lift of $\pi^{-1}\left[\left.[T M-\mathcal{L}]\right|_{\Gamma}-\left[N_{\Gamma}\right]\right]$ by the natural map $K^{0}\left(\Gamma^{\prime}, \Gamma^{\prime} \backslash \Sigma\right) \rightarrow K\left(\Gamma^{\prime}\right)$. We denote by $\eta=\eta(\mathcal{F}, \Gamma)$ this element in $K\left(\Gamma^{\prime}, \Gamma^{\prime} \backslash \Sigma^{\prime}\right)$, and denote by $\operatorname{GSV}(\mathcal{F}, \Gamma)=\operatorname{Res}(\eta(\mathcal{F}, \Gamma))$ its residue.

We also set, with the notation of $\S 2.2$,

$$
\operatorname{GSV}_{m_{\alpha}}(\mathcal{F}, \Gamma)=\operatorname{Res}_{m_{\alpha}}(\eta(\mathcal{F}, \Gamma)) \quad \text { and } \operatorname{GSV}_{h}(\mathcal{F}, \Gamma)=\operatorname{Res}_{h}(\eta(\mathcal{F}, \Gamma))
$$




\section{Cavalier and D. Lehmann}

Remarks.

(1) As $(* * *)$ is obtained by composition of the exact sequence $(* *)$ with $\ell$, we get

$$
\eta=\zeta+\xi \quad \text { in } K\left(\Gamma^{\prime}, \Gamma^{\prime} \backslash \Sigma^{\prime}\right) .
$$

(2) In the case $n=2$, and if $v$ is a local vector field on $M$ vanishing at $m$ and tangent to each $\Gamma_{h}$ through $m, \operatorname{GSV}_{m}(v, \Gamma)$ is equal to the GSV-index of $v$ at $m$ with respect to $\Gamma$ such as defined in [GSV91], hence the notation in higher dimension. The case of $\Gamma$ being a locally complete intersection in $M$ defined by a section (s-LCI) has already been defined in [LSS95] and [LS99]. The $K$-theoretical definition of GSV has already been given in [CL01] for $n=2$, and in [CLS05] in the general case.

(3) Be careful not to confuse $\operatorname{GSV}_{m_{\alpha}, h}\left(\mathcal{F}, \Gamma\right.$ ) (the contribution of the branch $\Gamma_{h}$ to the GSV-index at $m_{\alpha}$ with respect to $\Gamma$ ), with $\operatorname{GSV}_{m_{\alpha}}\left(\mathcal{F}, \Gamma_{h}\right)$ (the GSV-index at $m_{\alpha}$ with respect to $\Gamma_{h}$ ). See, for instance, [Suw95] in the case $n=2$.

Theorem 4.1. For any irreducible component $C$ of $\Gamma$, the following formulae hold:

$$
\left(c_{1}\left(\left[T M-N_{\Gamma}\right]\right) \frown[C]\right)+2 g_{C}-2=-\sum_{h \subset C} \mathcal{E}_{h}(\Gamma)
$$

and

$$
\left(c_{1}\left(\left[T M-N_{\Gamma}\right]\right) \frown[C]\right)-\left(c_{1}(\mathcal{L}) \frown[C]\right)=\sum_{h \subset C} \operatorname{GSV}_{h}(\Gamma) .
$$

Proof. Apply Lemma 2.3 to $\xi$ and to $\eta=\xi+\zeta$.

We want now to compute $\mathcal{E}_{h}(\Gamma)$. We can trivialize $T \Gamma^{\prime}$ by $\partial / \partial t$, and $T M$ by $\left(\partial / \partial x_{1}, \ldots, \partial / \partial x_{n}\right)$. Define in the same way $\beta_{1}(t)$ for $\lambda_{0} \circ q, \beta_{2}(t)$ for $\lambda_{1}$ and $\beta_{3}(t)$ for $\lambda_{2}$. With the notation of Theorem 2.4, take for $\psi_{0}$ the $1 \times 1$ matrix $\left(\varphi_{i}^{\prime}\right)$, the index $i$ being chosen such that $\varphi_{i}(t)$ is not constant near 0 . Define by induction, as in Theorem 2.4, $\psi_{1}=\psi_{1, i}, \psi_{2}=\psi_{2, i}$ and $\psi_{3}=\psi_{3, i}$ from this choice of $i$. Denoting by $\nu_{1, i}$ (respectively $\nu_{2, i}$, respectively $\nu_{3, i}$ ) the order $\nu$ (det $\psi_{1, i}(u)$ ) (respectively $\nu\left(\operatorname{det} \psi_{2, i}(u)\right)$, respectively $\left.\nu\left(\operatorname{det} \psi_{3, i}(u)\right)\right)$.

THEOREM 4.2. We have the following.

(i) With the notation above, the residue $\mathcal{E}_{h}(\Gamma)$ is given by the formula

$$
\mathcal{E}_{h}(\Gamma)=\nu_{1, i}-\nu_{2, i}+\nu_{3, i}-p_{i}+1,
$$

which is independent of the index $i$ such that $\varphi_{i}(t)$ is not constant along $\Gamma_{h}$.

(ii) The following inequality holds: $\mathrm{GSV}_{h}(\Gamma) \geqslant p_{i}-\nu_{1, i}+\nu_{2, i}-\nu_{3, i}$.

Proof. Apply Theorem 2.4 to $\xi$, and use the equality $\operatorname{GSV}_{h}(\mathcal{F}, \Gamma)=\mu_{m_{\alpha}}\left(\varphi^{-1} v, \Gamma_{h}\right)-\mathcal{E}_{h}(\Gamma)$.

In particular, if $\Gamma$ is a LCI locally defined near a point $m_{\alpha} \in \Gamma_{h}$ by the reduced equation $f=0$ with $f=\left(f_{1}, \ldots, f_{n-1}\right)$, assume $\mathcal{F}$ to be locally defined by the vector field $v=\sum_{i} A_{i}\left(\partial / \partial x_{n}\right)$, with respect to local coordinates $\left(x_{1}, \ldots, x_{n}\right)$ near $m_{\alpha}$.

Corollary 4.3. Assume that $\Gamma$ is a LCI. With the notation above, the residues $\mathcal{E}_{h}(\Gamma)$ and $\mathrm{GSV}_{h}\left(\mathcal{F}, \Gamma\right.$ ) are given, for any index $i$ such that $\varphi_{i}(t)$ (or, equivalently, $A_{i} \circ \varphi$ ) is not identically zero along $\Gamma_{h}$, by the formulae

$$
\mathcal{E}_{h}(\Gamma)=\nu_{i}(f)-p_{i}+1
$$

and

$$
\begin{aligned}
\operatorname{GSV}_{h}(\mathcal{F}, \Gamma) & =\nu\left(A_{i} \circ \varphi\right)-\nu_{i}(f), \\
& \geqslant p_{i}-\nu_{i}(f),
\end{aligned}
$$




\section{On the Poincaré inequality for One-Dimensional Foliations}

where $\nu_{i}(f)$ denotes the order of

$$
\left(\operatorname{det} \frac{D\left(f_{1}, \ldots, f_{n-1}\right)}{D\left(x_{1}, \ldots, \widehat{x_{i}}, \ldots, x_{n}\right)}\right) \circ \varphi
$$

Proof. In fact, in this case,

$$
\beta_{1}=\frac{D\left(f_{1}, \ldots, f_{n-1}\right)}{D\left(x_{1}, \ldots, x_{n}\right)}
$$

with obvious local trivializations of the bundles, while $\beta_{2}=\beta_{3}=0$.

Remark. While $\left[E_{0}-E_{1}+E_{2}-\hat{\pi}^{-1}(T M)+T \Gamma^{\prime}\right]$ and $\left[E_{0}-E_{1}+E_{2}\right]-\hat{\pi}^{-1}[T M-\mathcal{L}]$ do not depend in $K\left(\Gamma^{\prime}\right)$ on the resolution $(*)$ (as the same is true for $\left[E_{0}-E_{1}+E_{2}\right]$ after [AH61]), we have not been able to prove that it remains true for their lift $-\mathcal{E}(\Gamma)$ and $\operatorname{GSV}(\mathcal{F}, \Gamma)$ in $K\left(\Gamma^{\prime}, \Gamma^{\prime} \backslash \Sigma^{\prime}\right)$, unless $\Gamma$ is a LCI. Thus, all computations of residues in the non-LCI case are relative to a particular resolution. However, the sum of these residues does not depend on the resolution, once mapped into $H_{0}\left(\Gamma^{\prime}\right)$ by the natural map $H_{0}\left(\Sigma^{\prime}\right) \rightarrow H_{0}\left(\Gamma^{\prime}\right)$.

\section{Examples}

\subsection{Case $n=2$}

In the case of an algebraic foliation on $\mathbb{P}_{2}$, we get

$$
c_{1}\left(\left[T M-N_{\Gamma}-\mathcal{L}\right) \frown[C]=(d+2-\delta) \delta_{C} .\right.
$$

Proposition 5.1. If $\Gamma$ has an irreducible component $C$ such that all singularities of $\mathcal{F}$ which are in $C$ are non-dicritical, then $d+2-\delta \geqslant 0$.

Proof. In fact, the indices $\mathrm{GSV}_{m_{\alpha}}(\mathcal{F}, \Gamma)$ at a non-dicritical singularity $m_{\alpha}$ are all non-negatives after [Bru97], and the same is true for $\operatorname{GSV}_{h}(\mathcal{F}, \Gamma)$ after [CL01] if $\Gamma_{h}$ is included into $C$, for $C$ as in the statement of the proposition.

In particular, we recover the result of [Bru97, Car94] when $\Gamma$ has only non-dicritical singularities.

Case of smooth branches. Assume that $\Gamma_{h}$ is a smooth branch of $\Gamma$ through a singular point $m_{\alpha}$. Then, we can choose local coordinates $(x, y)$ such that $\Gamma_{h}$ has $y=0$ for an equation and parametrize $\Gamma_{h}$ by the map $\varphi: t \mapsto(x(t)=t, y(t) \equiv 0)$. There exists some holomorphic function $g \rightarrow U \rightarrow \mathbb{C}$, such that $f(x, y)=y g(x, y)$ and $g(x, 0)$ is not identically zero.

Lemma 5.2. The following formula holds: $\mathcal{E}_{h}(\Gamma)=\nu(g \circ \varphi)$.

Proof. Use Corollary 4.3 after observing that $f_{y}^{\prime}(x, 0)=g(x, 0)$.

In particular, call any singular point $m_{\alpha}$ with exactly $r$ local branches, all smooth, and having distinct tangents an 'elementary' $r$-multiple point of $\Gamma(r \geqslant 2)$. We then get the following.

Proposition 5.3. If $m_{\alpha}$ is an elementary $r$-multiple point of $\Gamma$ then, for any local branch through $m_{\alpha}$ and for any foliation $\mathcal{F}$, the following formulae hold:

$$
\begin{gathered}
\mathcal{E}_{h}(\Gamma)=r-1 \quad \text { and } \quad \operatorname{GSV}_{h}(\mathcal{F}, \Gamma) \geqslant-(r-2) . \\
\mathcal{E}_{m_{\alpha}}(\Gamma)=r(r-1) \quad \text { and } \quad \operatorname{GSV}_{m_{\alpha}}(\mathcal{F}, \Gamma) \geqslant-r(r-2)
\end{gathered}
$$

Proof. Under the given assumptions, the order of the function $g$ above $\Gamma_{h}$ is $r-1$, hence the result, using Lemma 5.2 and Corollary 4.3. 


\section{Cavalier and D. Lehmann}

Theorem 5.4. Let $\mathcal{F}$ be a foliation of degree $d$, leaving invariant an irreducible curve $\Gamma$ of degree $\delta$ in $\mathbb{P}_{2}$. Assume that $\Gamma$ has only elementary singularities, and let $n_{r}$ be the number of $r$-multiple points. Then, the following formulae hold:

$$
(d-1) \delta+2-2 g \geqslant \sum_{r \geqslant 2} n_{r} r, \quad \text { and } \quad(d+2-\delta) \delta \geqslant-\sum_{r \geqslant 2} n_{r} r(r-2) .
$$

More generally, if $\Gamma$ is reducible and has only elementary singularities, denote by $n_{r}(C)$ the total number of local branches included into some irreducible component $C$ through singular $r$-multiple points of $\Gamma$. Then, the following formulae hold:

$$
(d-1) \delta_{C}+2-2 g_{C} \geqslant \sum_{r \geqslant 2} n_{r}(C), \quad \text { and } \quad(d+2-\delta) \delta_{C} \geqslant-\sum_{r \geqslant 2} n_{r}(C)(r-2) .
$$

Proof. Combine Proposition 5.3 above with Theorems 3.1 and 4.1.

Corollary 5.5. If a curve $\Gamma$ has an irreducible component $C$ such that all singularities of $\Gamma$ which are in $C$ are nodal points of $\Gamma$, then the inequality $d+2-\delta \geqslant 0$ still holds.

In particular, we recover the result of [CL91, Soa01], when $\Gamma$ has only nodal singularities.

Example 5.6 (Reducible case). Let $\Gamma_{1}$ be a non-degenerate conic in $\mathbb{P}_{2}, m_{0}$ be a point of $\Gamma_{1}$, and $\left(\Delta_{i}\right)_{i}$ a family of $s-1$ projective straight lines $\Delta_{i}$ through $m_{0}$ in the plane $(s \geqslant 3)$, none of them being tangent to $\Gamma_{1}$. Taking for $\Gamma$ the union of this conic and of these straight lines, all singularities of $\Gamma$ are nodal (double point), except $m_{0}$ which is an $s$-uple point, and the degree of the curve is $s+1$. After Theorem 4.1, we get

$$
\begin{gathered}
d+1-s \geqslant-(s-2) \quad \text { with respect to } \Delta_{i}, \\
(d+1-s) 2 \geqslant-(s-2) \quad \text { with respect to the conic. }
\end{gathered}
$$

The strongest of these inequalities is of course the second, hence $d \geqslant s / 2$.

Other examples arise from [Lin02]. For instance, with homogeneous coordinates $(X, Y, Z)$ in $\mathbb{P}_{2}$, the set $\Gamma$ of the equation $\left(X^{3}-Z^{3}\right)\left(Y^{3}-Z^{3}\right)\left(X^{3}-Y^{3}\right)=0$ is the union of nine straight lines with 12 singularities (which are all elementary triple points): thus, we get $d \geqslant 3$; this proves that the family $\mathcal{F}_{\alpha}^{4}$ which leaves $\Gamma$ invariant does not have the minimal degree allowed by our formula. The image of these foliations by the map $S$ defined, for $Z \neq 0$, by $(x, y) \mapsto(x+y, x y)$ (with $x=X / Z, y=Y / Z$ ) is a family $\mathcal{F}_{\alpha}^{3}$ of foliations of degree 3. The image $S(\Gamma)$, preserved by the foliations $\mathcal{F}_{\alpha}^{3}$, is the union of two conics $C_{1}$ and $C_{2}$ (which are bitangent) and of three straight lines (which are tangent to $C_{1}$ and which intersect on $C_{2}$ ). The corollary of Theorem 3.1 then gives $d \geqslant 2$ when applied to one of the lines and $d \geqslant 3$ when applied to one of the conics; thus, $\mathcal{F}_{\alpha}^{3}$ here has the minimal degree authorized by our inequality. On these two examples, the quantity $d+2-\delta$ takes, respectively, the strictly negative values -3 and -2 : this proves a priori that the foliations $\mathcal{F}_{\alpha}^{4}$ and $\mathcal{F}_{\alpha}^{3}$ do have dicritical singularities.

\subsection{Higher dimension}

Theorem 5.7. Assume $\mathcal{F}$ to be an algebraic foliation of degree $d$ on $\mathbb{P}_{n}$, leaving invariant the complete intersection $\Gamma=\bigcap_{\lambda=1}^{n-1} S_{\lambda}$ of $n-1$ algebraic hypersurfaces $S_{\lambda}$. For any irreducible component $C$ of $\Gamma$, we get

$$
\left(d+n-\sum_{\lambda=1}^{n-1} \delta_{\lambda}\right) \delta_{C} \geqslant \mathcal{B}_{C}(\Gamma)-\mathcal{E}_{C}(\Gamma),
$$

where $\delta_{\lambda}$ and $\delta_{C}$ denote the degree of $S_{\lambda}$ and of $C$, respectively. 


\section{On the Poincaré inequality for one-Dimensional foliations}

Proof. Use Theorem 4.1, with $N_{\Gamma}=\bigoplus_{\lambda} \mathcal{O}\left(\delta_{\lambda}\right)$.

COROLlary 5.8. Under the assumptions of Theorem 5.7, assume moreover that $\Gamma$ has a smooth irreducible component $C$. Then we have the inequality

$$
\left(d+n-\sum_{\lambda=1}^{n-1} \delta_{\lambda}\right) \delta_{C} \geqslant \mathcal{B}_{C}(\Gamma) .
$$

Proof. In fact, in this case, $\mathcal{E}_{C}(\Gamma)=0$. As $\mathcal{B}_{C}(\Gamma) \geqslant 1$, we recover in particular the formula given in [Soa00], when $\Gamma$ is smooth.

Proposition 5.9. Assume that $m_{\alpha}$ has four branches, all smooth, with distinct tangents, and such that three of them are never coplanar. If $\Gamma$ is a LCI, then we have

$$
\mathcal{E}_{h}(\Gamma)=2, \quad \text { and } \operatorname{GSV}_{h}(\mathcal{F}, \Gamma) \geqslant-1 .
$$

Proof. Under the assumption, there exist local coordinates $(x, y, z)$ near $m_{\alpha}$, such that one branch is given by the local equations $(x=0, y=0)$, a second by $(x=0, z=0)$ and a third by $(y=0, z=0)$. The fourth branch, which may always be parametrized by $z$, has local equations $x-\varphi(z)=0, y-$ $\psi(z)=0$, with $\varphi(0)=\psi(0)=0, \varphi^{\prime}(0) \neq 0, \psi^{\prime}(0) \neq 0$. The curve $\Gamma$ is then locally defined by the equations $f=0$, with $f_{1}(x, y, z)=x(y-\psi(z))$ and $f_{2}(x, y, z)=y(x-\varphi(z))=0$. The jacobian matrix is

$$
\frac{D\left(f_{1}, f_{2}\right)}{D(x, y, z)}=\left(\begin{array}{ccc}
y-\psi(z) & x & -x \psi^{\prime}(z) \\
y & x-\varphi(z) & -y \varphi^{\prime}(z)
\end{array}\right) .
$$

Using Theorem 4.2 along the branch $y=z=0$, we get $\mathcal{E}_{h}(\Gamma)=2$. All branches in fact playing the same role, the same formula is true for the three other branches.

Example 5.10 (Not a LCI). Let $\Gamma$ be the rational quintic parametrized by the map

$$
[u, v] \mapsto\left[X(u, v)=u^{3} v^{2}, Y(u, v)=u^{4} v, Z(u, v)=u^{5}, T(u, v)=v^{5}\right]
$$

from $\mathbb{P}_{1}$ (with homogeneous coordinates $[u, v]$ ) into $\mathbb{P}_{3}$ (with homogeneous coordinates $[X, Y, Z, T]$ ). It has only the origin for singular point with one local branch at this point, hence $\mathcal{B}(\Gamma)=1$. According to [CLS04], $c_{1}\left(N_{\Gamma}\right) \frown[\Gamma]=21$, hence $\mathcal{E}(\Gamma)=3$. We get $d \geqslant 1$ (we can also use Theorem 3.1, with $g=0$ ). In fact, $\Gamma$ is invariant by the foliation of degree 1 defined by the vector field $3 x(\partial / \partial x)+4 y(\partial / \partial y)+5 z(\partial / \partial z)$ in $\mathbb{C}^{3}$ : thus, the lower bound for $d$ is reached. Note that this foliation has dicritical singularities. It can be shown that the minimal degree of the foliations without dicritical singularities leaving this quintic invariant is 2 .

\section{ACKNOWLEDGEMENT}

We thank M. Soares for helpful correspondence, as well as the referee for his suggestions.

\section{REFERENCES}

Ati64 M. F. Atiyah, K-theory, Notes by D. W. Anderson (Harvard University, Cambridge, MA, 1964).

AH61 M. F. Atiyah and F. Hirzebruch, Analytic cycles on complex manifolds, Topology 1 (1961), 25-45.

Bru97 M. Brunella, Some remarks on indices of holomorphic vector fields, Publ. Mat. 41 (1997), $527-544$.

CC97 A. Campillo and M. Carnicer, Proximity inequalities and bounds for the degree of invariant curves by foliations of $\mathbb{P}_{\mathbb{C}}^{2}$, Trans. Amer. Math. Soc. 349 (1997), 2211-2228.

Car94 M. Carnicer, The Poincaré problem in the nondicritical case, Ann. of Math. (2) 140 (1994), 289-294. 


\section{On the Poincaré INEQUALITY FOR ONE-DIMENSIONAL FOLIATIONS}

CL01 V. Cavalier and D. Lehmann, Localisation des résidus de Baum-Bott, courbes généralisées et K-théorie. Première partie: Feuilletages dans $\mathbb{C}^{2}$, Comment. Math. Helv. 76 (2001), 1-19.

CLS04 V. Cavalier, D. Lehmann and M. G. Soares, Classes de Chern des ensembles analytiques, C. R. Acad. Sci. Paris Ser. I 338 (2004), 879-884.

CLS05 V. Cavalier, D. Lehmann and M. G. Soares, Chern classes of analytical sets and applications, $K$-theory 34 (2005), 35-69.

CL91 D. Cerveau and A. Lins Neto, Holomorphic foliations in $\mathrm{CP}(2)$ having an invariant algebraic curve, Ann. Inst. Fourier (Grenoble) 41 (1991), 883-903.

EK03 E. Esteves and S. Kleiman, Bounds on leaves of one-dimensional foliations, Bull. Braz. Mat. Soc. (NS) 34 (2003), 145-169.

GSV91 X. Gomez-Mont, J. Seade and A. Verjovski, The index of a holomorphic flow with an isolated singularity, Math. Ann. 291 (1991), 737-751.

LS95 D. Lehmann and T. Suwa, Residues of holomorphic vector fields relative to singular invariant subvarieties, J. Differential Geom. 42 (1995), 367-384.

LS99 D. Lehmann and T. Suwa, Generalization of variations and Baum-Bott residues for holomorphic foliations on singular varieties, Internat. J. Math. 10 (1999), 367-384.

LSS95 D. Lehmann, M. G. Soares and T. Suwa, On the index of a holomorphic vector field tangent to a singular variety, Bol. Soc. Bras. Mat. 26 (1995), 183-199.

Lin02 A. Lins Neto, Some examples for Poincaré and Painlevé problems, Ann. Sci. Ecole Norm. Sup. (4) 35 (2002), 231-266.

Soa97 M. G. Soares, The Poincaré problem for hypersurfaces invariant by one-dimensional foliations, Invent. Math. 128 (1997), 495-500.

Soa00 M. G. Soares, Projective varieties invariant by one-dimensional foliations, Ann. of Math. (2) 152 (2000), 369-382.

Soa01 M. G. Soares, On the geometry of Poincaré's problem for one-dimensional projective foliations, An. Acad. Brasil. Ciênc. 73 (2001), 475-482.

Suw95 T. Suwa, Indices of holomorphic vector fields relative to invariant curves on surfaces, Proc. Amer. Math. Soc. 123 (1995), 2989-2997.

Vincent Cavalier cavalier@math.univ-montp2.fr Département des Sciences Mathématiques, CP 051, Université de Montpellier II, Place Eugène Bataillon, F-34095 Montpellier cedex 5, France

Daniel Lehmann lehmann@math.univ-montp2.fr

Département des Sciences Mathématiques, CP 051, Université de Montpellier II, Place Eugène Bataillon, F-34095 Montpellier cedex 5, France 